\title{
RELAÇÃO ENTRE O CONHECIMENTO E A REALIZAÇÃO DO EXAME PAPANICOLAOU EM UNIVERSITÁRIAS DE MEDICINA E PEDAGOGIA
}

Juliana Loiara Roxinol, Camila Souza Vilela, Gabriella Veloso Santana, Isadora Daleffi Zocoler, Nathalye Fernanda Pedroso Dircksen, Emanuele Moraes Mello

Universidade do Oeste Paulista - UNOESTE, Faculdade de Medicina, Presidente Prudente, SP. E-mail: emanuelemed@yahoo.com.br.

\section{RESUMO}

Estudo transversal com 319 acadêmicas de Pedagogia e Medicina composto de questionário, adaptado de Cruz, com 15 perguntas de autopreenchimento englobando os dados socioeconômicos e conhecimentos relativos ao Papanicolaou. Para as análises estatísticas utilizouse programa Bioestat 5.3. Empregou-se o teste do qui-quadrado, teste $\mathrm{G}$ ou teste exato de Fisher. Nível alfa para todos os testes foi 0,05 . As acadêmicas, em sua maioria (88,05\%), conhecem o exame $(p=0,2541)$ e sabem sua finalidade $(p=0,2139)$. Porém, a cobertura de realização do mesmo é insatisfatória (40\% nunca realizaram, $p=0,7821)$. Houve discrepância ao analisar dados socioeconômicos entre os cursos ( $p<0,05$ para todos) e o fato das acadêmicas de Medicina utilizarem mais métodos preventivos: anticoncepcional oral $(p=0,0008)$ e os de barreira $(p=0,0026)$. O conhecimento acerca do exame Papanicolaou não dependeu do curso e a informação a respeito desse não é fator determinante para realizá-lo visto que a cobertura de procedimentos em ambas as graduações é insatisfatória.

Palavras-chave: Pedagogia, Medicina, Papanicolaou, anticoncepcional oral, cobertura.

\begin{abstract}
Cross-sectional study with 319 Pedagogy and Medicine students, composed of a questionnaire, adapted from Cruz, with 15 self-filling questions encompassing socioeconomic and Pap smear knowledge. For statistical analysis, the program Bioestat 5.3 was used and the chi-square test, Gtest or Fisher's exact test. Alpha level for all tests was 0.05 . The majority of the students $(88.05 \%)$ know the exam $(p=0.2541)$ and their purpose $(p=0.2139)$. However, coverage of the same is unsatisfactory (40\% never performed, $p=0.7821)$. There's a discrepancy when analyzing socioeconomic data between the courses $(p<0.05$ for all) and the fact that medical students used more preventive methods: oral contraceptives $(p=0.0008)$ and barrier method $(p=0.0026)$. Knowledge about the Pap smear hasn't depended on the course, and the information about it is not a determining factor to carry it out since coverage of procedures in both grades is unsatisfactory.
\end{abstract}

Keywords: Pedadogy, Medicine, Pap smear, oral contraceptives, coverage. 


\section{INTRODUÇÃO}

O câncer uterino, também conhecido como cervical, é a segunda neoplasia mais incidente na população feminina mundial, levando cerca de 275 mil mulheres à óbito por ano (RIBEIRO et al., 2015). As taxas de mortalidade por câncer de colo do útero, no Brasil, em 2013 somam o valor de 5.430 óbitos. (INCA, 2015). Para o ano de 2016 estima-se que 16.340 novos casos sejam detectados (INCA, 2015).

O Papanicolaou, também conhecido como citologia oncológica é o método mais eficaz e eficiente para o diagnóstico precoce do câncer de colo de útero (BRASIL, 1997). O exame consiste no estudo de células do colo uterino, onde se é possível detectar a presença de células neoplásicas a partir do esfregaço do epitélio vaginal. A coleta deve ser realizada por um médico ou enfermeiro. Antes da realização, a cliente não deve manter relações sexuais, não utilizar duchas ou medicamentos vaginais por um período de 48 horas e não estar menstruada. Se realizado por meio de uma técnica adequada o procedimento é simples, rápido e causa no máximo um pequeno desconforto (BRASIL, 2011).

De acordo com PICHON-RIVIÈRE (1988) "o objeto de formação do profissional deve instrumentar o sujeito para uma prática de transformação de si, dos outros e do contexto em que estão inseridos" e, ainda ressalta que "a aprendizagem centrada nos processos grupais coloca em evidência a possibilidade de uma nova elaboração de conhecimento, de integração e de questionamentos acerca de si e dos outros". Baseado nisso, a organização curricular do curso de Medicina possibilita que as acadêmicas entrem em contato com metodologias ativas de ensino e aprendizagem. Através dessa participação pretende-se melhorar a qualidade de vida das pessoas na comunidade onde estão incluídas.

Enquanto isso é sabido que a formação no curso de pedagogia deve estar relacionada com a realidade e o processo educativo a ser desenvolvido após a graduação, de forma que ao final do curso os profissionais estejam aptos para comprometer-se com a transformação da sociedade. Neste sentido, o pedagogo não exercerá apenas o papel de executor de diretrizes e princípios educacionais, mas também o de um disseminador da Educação para uma sociedade em constantes transformações (MINISTÉRIO DA EDUCAÇÃO, 2002).

\section{METODOLOGIA}

Estudo individualizado do tipo transversal, aprovado pelo Comitê de Ética e Pesquisa (protocolo n.3367) e Plataforma Brasil (CAAE n. 58181916.0.0000.5515), realizado na Universidade do Oeste Paulista (UNOESTE). A amostra foi composta por alunas do primeiro e último ano dos cursos de graduação em medicina e pedagogia, abordadas aleatoriamente. Realizada entrevista, que se baseou em um questionário, adaptado de Cruz (2013) composto por 15 perguntas de autopreenchimento que englobam dados socioeconômicos e conhecimentos relativos ao exame preventivo. As análises estatísticas foram realizadas no programa Bioestat 5.3; sendo os testes: qui-quadrado, teste $\mathrm{G}$ e teste exato de Fisher. O nível alfa foi de 0,05 (AYRES et al.; 2007).

\section{RESULTADOS}

Participaram desse estudo 185 alunas do curso de medicina e 134 do curso de pedagogia. Com relação aos dados socioeconômicos, houve predominância de faixa etária superior a 25 anos tanto na medicina $(85,4 \%)$, quanto na pedagogia $(60,44 \%)$; já com relação ao estado civil, houve predominância de estudantes solteiras na medicina $(95,14 \%)$ e de casadas na pedagogia $(59,70 \%)$. Quanto à atividade remunerada, a mesma está presente em $95,13 \%$ das estudantes de medicina e $67,13 \%$ das de pedagogia, porém diferiu quanto à renda familiar, sendo em $82,16 \%$ superior a 2,1 salários mínimos nas estudantes de medicina e em $54,47 \%$ das estudantes de pedagogia entre 1,1 a 2 salários mínimos. Houve uma predominância de estudantes de raça branca e católicas em ambos os cursos, sendo respectivamente $84,37 \%$ e $72,43 \%$ nas estudantes de medicina, e de 
$52,23 \%$ e $61,94 \%$ nas de pedagogia. Para esses dados, todos foram estatisticamente significativos $(p<0,0001)$, incluindo a religião $(p=0,0163)$.

Com relação ao questionário, no quesito sobre o conhecimento do exame preventivo e a sua finalidade, ou seja, a prevenção; a prevalência das respostas entre as acadêmicas de medicina e pedagogia foram similares, mostrando que pelo menos $88,05 \%$ dessas possui essa informação $(p=0,2541)$. Quanto à realização do exame, também foram encontrados dados semelhantes entre os cursos, mostrando que 60\% dessas afirmaram terem feito o exame em até 2 anos. Em relação ao local de realização do Papanicolaou e retorno para avaliar resultado do mesmo, 54,60\% procuram por serviço particular e $49,73 \%$ retornam para buscar o exame dentre as alunas de medicina e $32,08 \% / 56,71 \%$ da pedagogia, respectivamente, fazem o procedimento em local privado e dão seguimento. Aproximadamente $40 \%$ das entrevistadas relataram nunca ter feito o exame, sendo a justificativa predominante encontrada ser por motivos diversos, que não estão relacionados com medo, vergonha ou falta de tempo. Porém, a maioria afirmou conhecer as recomendações previas para a realização do exame (78,91\% - medicina; 76,87\% - pedagogia) e as complicações da não realização do mesmo (94,59\% - medicina; 81,34\% - pedagogia).

Houve uma predominância da confirmação do uso regular de anticoncepcional em 71,89\% x 53,73\% das acadêmicas da medicina e pedagogia respectivamente, de caráter significativo ( $p=0,0008$ ) (Tabela 1). Quanto ao uso de contraceptivos de barreira (Tabela 2), 45,40\% das acadêmicas de medicina responderam que fazem o uso, porém diferiu das acadêmicas de pedagogia, onde $44,79 \%$ responderam não fazer uso do a mesmo $(p=0,0026)$. Com relação à prática de atividade sexual, as acadêmicas que afirmam serem sexualmente ativa e com um único parceiro sexual, são respectivamente, $64,32 \% / 52,43 \%$ da medicina e $70,89 \% / 72,38 \%$ da pedagogia. Ainda, quanto ao número de parceiros demonstrou-se uma amostra significativa ( $p=0,0034$ ). Além disso, $98,37 \%$ das acadêmicas de medicina e $96,26 \%$ das de pedagogia afirmaram nunca terem tido uma DST. A idade da primeira relação sexual até 18 anos, foi de $49,72 \%$ nas acadêmicas da medicina e $51,49 \%$ das de pedagogia. Dentre as que afirmaram nunca terem tido relação sexual, $22,16 \%$ são da medicina e $16,41 \%$ da pedagogia. Já com relação a maternidade, 5,41\% das acadêmicas da medicina e 34,33\% das da pedagogia afirmaram terem filhos, 0 que se demonstrou uma proporção significativa $(p<0,001)$. 
Tabela 1. Valores absolutos ( $\mathrm{n}$ ) e percentuais (\%) das respostas ao questionário das participantes da pesquisa de acordo com o uso regular de anticoncepcional, independentemente do curso e individualizado por curso de medicina e pedagogia.

\begin{tabular}{|c|c|c|c|c|}
\hline \multicolumn{5}{|c|}{ Medicina e pedagogia } \\
\hline \multirow{2}{*}{$\begin{array}{l}\text { Uso regular de } \\
\text { anticoncepcional }\end{array}$} & \multicolumn{2}{|c|}{ Conhece o exame } & \multicolumn{2}{|c|}{ Não conhece o exame } \\
\hline & $\mathbf{N}$ & $\%$ & $\mathbf{N}$ & $\%$ \\
\hline Sim & 193 & 94,15 & 12 & 5,85 \\
\hline Não & 94 & 82,46 & 20 & 17,54 \\
\hline Valor de $p$ & & & & \\
\hline \multicolumn{5}{|c|}{ Medicina } \\
\hline \multirow{2}{*}{$\begin{array}{l}\text { Uso regular de } \\
\text { anticoncepcional }\end{array}$} & \multicolumn{2}{|c|}{ Conhece o exame } & \multicolumn{2}{|c|}{ Não conhece o exame } \\
\hline & $\mathbf{N}$ & $\%$ & $\mathbf{N}$ & $\%$ \\
\hline Sim & 127 & 95,49 & 6 & 4,51 \\
\hline Não & 43 & 82,69 & 9 & 17,31 \\
\hline Valor de $\mathrm{p}$ & & & & \\
\hline \multicolumn{5}{|c|}{ Pedagogia } \\
\hline \multirow{2}{*}{$\begin{array}{l}\text { Uso regular de } \\
\text { anticoncepcional }\end{array}$} & \multicolumn{2}{|c|}{ Conhece o exame } & \multicolumn{2}{|c|}{ Não conhece o exame } \\
\hline & $\mathbf{N}$ & $\%$ & $\mathbf{N}$ & $\%$ \\
\hline Sim & 66 & 91,67 & 6 & 8,33 \\
\hline Não & 51 & 82,26 & 11 & 17,74 \\
\hline Valor de $p$ & \multicolumn{4}{|c|}{0,1028} \\
\hline
\end{tabular}


Tabela 2. Valores absolutos ( $\mathrm{n}$ ) e percentuais (\%) das respostas ao questionário das participantes da pesquisa de acordo com o uso de contraceptivo de barreira, independentemente do curso e individualizado por curso de medicina e pedagogia.

\begin{tabular}{|c|c|c|c|c|}
\hline \multicolumn{5}{|c|}{ Medicina e pedagogia } \\
\hline \multirow{2}{*}{$\begin{array}{l}\text { Faz uso de contraceptivos } \\
\text { de barreira na relação } \\
\text { sexual (camisinha e/ou } \\
\text { diaframa)? }\end{array}$} & \multicolumn{2}{|c|}{ Conhece o exame } & \multicolumn{2}{|c|}{ Não conhece o exame } \\
\hline & $\mathbf{N}$ & $\%$ & $\mathbf{N}$ & $\%$ \\
\hline Sim & 118 & 90,77 & 12 & 9,23 \\
\hline Não & 169 & 89,42 & 20 & 10,58 \\
\hline Valor de $p$ & \multicolumn{4}{|c|}{0,6930} \\
\hline \multicolumn{5}{|c|}{ Medicina } \\
\hline \multirow{2}{*}{$\begin{array}{l}\text { Faz uso de contraceptivos } \\
\text { de barreira na relação } \\
\text { sexual (camisinha e/ou } \\
\text { diaframa)? }\end{array}$} & \multicolumn{2}{|c|}{ Conhece o exame } & \multicolumn{2}{|c|}{ Não conhece o exame } \\
\hline & $\mathbf{N}$ & $\%$ & $\mathbf{N}$ & $\%$ \\
\hline Sim & 79 & 95,18 & 4 & 4,82 \\
\hline Não & 91 & 89,22 & 11 & 10,78 \\
\hline Valor de $p$ & \multicolumn{4}{|c|}{0,1125} \\
\hline \multicolumn{5}{|c|}{ Pedagogia } \\
\hline \multirow{2}{*}{$\begin{array}{l}\text { Faz uso de contraceptivos } \\
\text { de barreira na relação } \\
\text { sexual (camisinha e/ou } \\
\text { diaframa)? }\end{array}$} & \multicolumn{2}{|c|}{ Conhece o exame } & \multicolumn{2}{|c|}{ Não conhece o exame } \\
\hline & $\mathbf{N}$ & $\%$ & $\mathbf{N}$ & $\%$ \\
\hline Sim & 39 & 90,70 & 4 & 9,30 \\
\hline Não & 78 & 85,71 & 13 & 14,29 \\
\hline Valor de $p$ & & & 3049 & \\
\hline
\end{tabular}

Comparando-se a faixa etária de ambos os cursos (de maneira isolada) ao nível de conhecimento do exame tem-se que quanto maior a idade, maior o nível de conhecimento sendo $100 \%$ nas acadêmicas de medicina e $63,62 \%$ entre as de pedagogia (Tabela 3). Em um panorama geral, 95,95\% acima dos 25 anos conhecem o Papanicolaou enquanto 88,16\% com menos de 25 anos tem esse conhecimento $(p=0,0331)$ demonstrando significância estatística.

Apesar da discrepância entre o nível econômico de ambos os cursos (Tabela 4), não houve significância estatística ao se levar em consideração o conhecimento que as alunas possuem do exame

$(p=0,2343)$. 
Tabela 3. Valores absolutos (n) e percentuais (\%) das respostas ao questionário das participantes da pesquisa de acordo com a faixa etária, independentemente do curso e individualizado por curso de medicina e pedagogia.

\begin{tabular}{|c|c|c|c|c|}
\hline \multicolumn{5}{|c|}{ Medicina e pedagogia } \\
\hline \multirow[t]{2}{*}{ Faixa etária } & \multicolumn{2}{|c|}{ Conhece o exame } & \multicolumn{2}{|c|}{ Não conhece o exame } \\
\hline & $\mathbf{N}$ & $\%$ & $\mathbf{N}$ & $\%$ \\
\hline Até 25 anos & 216 & 88,16 & 29 & 11,84 \\
\hline Acima de 25 anos & 71 & 95,95 & 3 & 4,05 \\
\hline Valor de $p$ & & & & \\
\hline \multicolumn{5}{|c|}{ Medicina } \\
\hline \multirow[t]{2}{*}{ Faixa etária } & \multicolumn{2}{|c|}{ Conhece o exame } & \multicolumn{2}{|c|}{ Não conhece o exame } \\
\hline & $n$ & $\%$ & $n$ & $\%$ \\
\hline Até 25 anos & 143 & 90,51 & 15 & 9,49 \\
\hline Acima de 25 anos & 27 & 100,00 & 0 & 0,00 \\
\hline Valor de $p$ & & & & \\
\hline \multicolumn{5}{|c|}{ Pedagogia } \\
\hline \multirow[t]{2}{*}{ Faixa etária } & \multicolumn{2}{|c|}{ Conhece o exame } & \multicolumn{2}{|c|}{ Não conhece o exame } \\
\hline & $n$ & $\%$ & $\mathbf{n}$ & $\%$ \\
\hline Até 25 anos & 73 & 83,91 & 14 & 16,09 \\
\hline Acima de 25 anos & 44 & 93,62 & 3 & 6,38 \\
\hline Valor de $\mathrm{p}$ & & & & \\
\hline
\end{tabular}


Tabela 4. Valores absolutos ( $\mathrm{n}$ ) e percentuais (\%) das respostas ao questionário das participantes da pesquisa de acordo com a renda familiar, independentemente do curso e individualizado por curso de medicina e pedagogia.

\begin{tabular}{|c|c|c|c|c|}
\hline \multicolumn{5}{|c|}{ Medicina e pedagogia } \\
\hline \multirow{2}{*}{$\begin{array}{l}\text { Renda familiar } \\
\text { (salários } \\
\text { mínimos) }\end{array}$} & \multicolumn{2}{|c|}{ Conhece o exame } & \multicolumn{2}{|c|}{ Não conhece o exame } \\
\hline & $\mathrm{n}$ & $\%$ & $n$ & $\%$ \\
\hline Até 1 & 19 & 5,95 & 3 & 0,94 \\
\hline De 1,1 a 2 & 162 & 50,78 & 13 & 4,07 \\
\hline Superior a 2,1 & 106 & 33,22 & 16 & 5,04 \\
\hline Valor de $p$ & & & & \\
\hline \multicolumn{5}{|c|}{ Medicina } \\
\hline \multirow{2}{*}{$\begin{array}{l}\text { Renda familiar } \\
\text { (salários } \\
\text { mínimos) }\end{array}$} & \multicolumn{2}{|c|}{ Conhece o exame } & \multicolumn{2}{|c|}{ Não conhece o exame } \\
\hline & $n$ & $\%$ & $n$ & $\%$ \\
\hline Até 1 & 2 & 1,08 & 0 & 0 \\
\hline De 1,1 a 2 & 96 & 51,89 & 3 & 1,62 \\
\hline Superior a 2,1 & 72 & 38,91 & 12 & 6,5 \\
\hline Valor de $\mathrm{p}$ & & & & \\
\hline \multicolumn{5}{|c|}{ Pedagogia } \\
\hline \multirow{2}{*}{$\begin{array}{l}\text { Renda familiar } \\
\text { (salários } \\
\text { mínimos) }\end{array}$} & \multicolumn{2}{|c|}{ Conhece o exame } & \multicolumn{2}{|c|}{ Não conhece o exame } \\
\hline & $\mathbf{n}$ & $\%$ & $n$ & $\%$ \\
\hline Até 1 & 17 & 12,68 & 3 & 2,23 \\
\hline De 1,1 a 2 & 66 & 49,25 & 10 & 7,46 \\
\hline Superior a 2,1 & 34 & 25,37 & 4 & 3,01 \\
\hline Valor de $\mathrm{p}$ & & & & \\
\hline
\end{tabular}

\section{DISCUSSÃO}

O exame Papanicolaou deveria ser do conhecimento de todas as mulheres, visto que é um meio de prevenção para evitar o desenvolvimento de uma patologia como o câncer de colo uterino. De tal modo, analisando-se o questionário de autopreenchimento das acadêmicas de Medicina e Pedagogia, constou-se que $90,28 \%$ conhecem o exame. $(p=0,2541)$ e em ambos os grupos a maioria sabe a finalidade do mesmo, demonstrando que o conhecimento delas independe do curso.

Desse modo, as acadêmicas de Medicina demonstraram que como futuras profissionais de saúde estão aptas a orientar as pacientes que estiverem sob seus cuidados, bem como as profissionais de pedagogia que têm o papel de disseminar informações importantes à sociedade, inclusive no aspecto de saúde.

No que diz respeito ao autocuidado das acadêmicas, os resultados demontraram que a cobertura nos grupos é insatisfatória. Avaliou-se que 40,12\% de todas as acadêmicas nunca realizaram o exame, demonstrando significância estatística. Já comparando-se um grupo de medicina com a pedagogia viu-se que a diferença em termos percentuais é quase insignificante. $E$, mesmo não realizando o procedimento, elas alegaram saber as consequências e possíveis complicações de não o fazer. Enquanto isso, a razão para não realização do exame ficou definido como outros motivos além dos fatores constrangimento, falta de tempo ou medo para ambos os cursos.

Dentre os fatores de risco para desenvolvimento de câncer colo uterino estão idade precoce para relações sexuais (BURD, 2003), uso de anticoncepcionais orais (GROSS \& BARRASCO, 
1999), múltiplos parceiros sexuais, o não uso de métodos de barreira que ajudam a evitar doenças sexualmente transmissíveis (DSTs) visto que o vírus HPV, maior precursor de neoplasia de colo uterino é transmitido via sexual (SANTOS et al., 2010/2011) e a quantidade de filhos (MUÑOZ et al.; 2002). Ao analisar o questionários, viu-se que as acadêmicas de medicina utilizam mais anticoncepcionais orais do que as alunas de pedagogia, bem como utilizam mais métodos de barreira. No entanto, possuem múltiplos parceiros em percentual maior. As discentes de pedagogia possuem mais filhos do que as de medicina. Em ambos os cursos, a maioria é sexualmente ativa, iniciou a vida sexual antes dos 18 anos e referiu não ter desenvolvido DST em algum momento.

Os fatores socioeconômicos demonstraram uma discrepância importante entre os cursos, como o esperado. A maioria das acadêmicas de medicina são solteiras $(95,14 \%)$, enquanto as de pedagogia são em maior parte casadas $(59,70 \%)$. Essas exercem mais atividade remunerada comparada àquelas, sendo um percentual de $32,84 \%$. No entanto, a renda familiar é maior nas futuras profissionais de saúde, onde predomina-se o valor de acima de 2 salários mínimos.

Com relação ao conhecimento efetivo do exame, as que não o conhecem tendem a ser as mais jovens (até 25 anos). Dado interessante foi que a renda familiar não foi fator determinante para conhecer o Papanicolaou, as que estavam entre 1,1 e 2 salários mínimos demonstraram estar mais atualizadas do que as com valor superior a 2 salários mínimos. $O$ uso de anticoncepcional oral é maior nas que conhecem, porém, o método de barreiras já não é utilizado em maioria dentre esse grupo que detém informação.

\section{CONCLUSÃO}

No estudo foi observado que a maioria das estudantes de Medicina e Pedagogia estão preparadas para repassarem seus conhecimentos acerca do exame em suas respectivas áreas de atuação. Entretanto, foi observado que apesar de conhecer e saberem a finalidade do exame Papanicolaou, as mesmas não aderem à sua realização em prol da sua própria saúde, independentemente do curso ou das condições socioeconômicas. Visto isso, é fundamental que a formação destas profissionais deva ser instrumento da educação permanente não só dos demais mas também de si mesmas, de forma que sejam estimuladas a realizar o exame e assim prevenir possíveis agravos futuros por meio da detecção precoce de câncer uterino, promovendo assim mais saúde e qualidade de vida para as mesmas.

\section{REFERÊNCIAS}

1. AYRES, M.; AYRES JR, M.; AYRES, D. L.; SANTOS, A. S. dos. BIOESTAT - Aplicações estatísticas nas áreas das Ciências Bio-Médicas. 5. ed. Belém - Pará, 2007.

2. BRASIL. Ministério da saúde. Secretaria Nacional de Assistência a Saúde Instituto Nacional de Câncer. Coordenação Nacional de Controle do Tabagismo, Prevenção e Vigilância do Câncer (Conprev). Falando sobre câncer e seus fatores de risco. Ed. 2 Rio de Janeiro: Instituto Nacional de câncer, 1997.

3. BRASIL. Ministério da Saúde. Exame preventivo do câncer de colo uterino (Papanicolau); julho. 2011.

4. BURD, M. E. Human Papillomavirus and Cervical Cancer. Revista Clinical Microbiology Reviews, v. 16, n. 1, p. 1-17, jan/2003.

5. CRUZ, D. E. da; JARDIM, D. P. Adolescência e Papanicolau: conhecimento e prática. Revista Adolescência \& Saúde, v. 10, supl. 1, p. 34-42, abril/2013. 
6. FRIGO, L. F.; ZAMBARDA, S. O.; Câncer do colo de útero: efeitos do tratamento cervical. Revista Cinergis, Santa Cruz do Sul , v. 16, n. 3, p. 164-168, Jul./Set. 2015.

7. GROSS G. E.; BARRASCO R. Infeç̧ão por Papilomavírus Humano; Atlas clínico de HPV, Porto Alegre: Artes Médicas Sul Ltda, 1999.

8. INCA. Instituto Nacional de Câncer José Alencar Gomes da Silva. Colo do útero. Disponível em: <http://www2.inca.gov.br/wps/wcm/connect/tiposdecancer/site/home/colo_utero>. Acesso em: 22 de fevereiro de 2016.

9. INCA. Instituto Nacional de Câncer (Brasil). Estimativa 2016. Incidência do Câncer no Brasil. Rio de Janeiro: INCA, $2015 . \quad$ Disponível em: <http://www2.inca.gov.br/wps/wcm/connect/acoes_programas/site/home/nobrasil/programa_n acional_controle_cancer_colo_utero/conceito_magnitude>. Acesso em: 04 de maio de 2016.

10. MINISTÉRIO DA EDUCAÇÃO. Conselho Nacional de Educação. Resolução CNE/CP1, de 18 de fevereiro de 2002.

11. MUÑOZ, N.; FRACESCHI, S.; BOSETTI, C.; MORENO, V.; HERRERO, R.; SMITH, J. S.; SHAH, K. V.; MEIJER, C. J. L. M.; BOSCH, F. X. Role of parity and Human Papillomavirus in cervical cancer: the IARC multicentric case-control study. Medical Journal The Lancet, v. 359, n. 9312, p. 1093-110, Março/2002.

12. MUÑOZ, N.; BOSCH, F. X.; SANJOSÉ, S. de.; HERRERO, R.; CASTELLSAGUÉ, X.; SHAH, K. V.; SNIJDERS, P. J. F.; MEIJER, C. J. L. M. Epidemiologic classification of human papillomavirus types associated with cervical cancer. The New England Journal of Medicine, v. 348, n. 6, p. 518-527, Fev/2003.

13. PICHON-RIVIÈRE, E. Processo Grupal. São Paulo: Martins Fontes, 1988.

14. RIBEIRO, J.; SILVA, A.; CAMPELO, V.; SANTOS, S.; COÊLHO, D. Perfil sociodemográfico e clínico de mulheres com câncer do colo do útero em uma cidade do Nordeste. Revista Eletrônica Gestão e Saúde, , v. 6, n. 2, p. 1367-1381, jan. 2015. Disponível em: <http://gestaoesaude.unb.br/index.php/gestaoesaude/article/view/952>. Acesso em: 13 Fev. 2016.

15. SANTOS, I. M.; MAIORAL, M. F.; HAAS, P. Infecção por HPV em homens: Importância na transmissão, tratamento e prevenção do vírus. Revista Estudos de Biologia, v. 32, n. 76/81, p. 111118, Jan./Dez. 2011.

16. SILVA, E. C. A.; DIAS, M. P.; FERNANDES, C. K.; NOGUEIRA, D. S.; BARROS, E. J.; MOTA, R. de. M.; OLIVEIRA, V. C. de. C.; MENDONÇA, B. de. O. M.; Conhecimento das mulheres de 18 a 50 anos de idade sobre a importância do exame Papanicolaou na prevenção do câncer de colo uterino no município de Turvânia-GO. Revista Faculdade Montes Belos (FMB), v. 8, n. 4, p. 99-122, 2015. 